\title{
A chemical screen for suppressors of the avrRpm1-RPM1- dependent hypersensitive cell death response in Arabidopsis thaliana
}

\author{
Mario Serrano • David A. Hubert • Jeffery L. Dangl • \\ Paul Schulze-Lefert $\cdot$ Erich Kombrink
}

Received: 24 August 2009/ Accepted: 18 January 2010/Published online: 7 February 2010

(C) The Author(s) 2010. This article is published with open access at Springerlink.com

\begin{abstract}
Arabidopsis thaliana RPMI encodes an intracellular immune sensor that conditions disease resistance to Pseudomonas syringae expressing the type III effector protein AvrRpm1. Conditional expression of this type III effector in a transgenic line carrying avrRpml under the control of a steroid-inducible promoter results in RPM1dependent cell death that resembles the cell death response of the incompatible RPM1-avrRpml plant-bacterium interaction. This line was previously used in a genetic screen, which revealed two genes that likely function in the folding of preactivation RPM1. We established a chemical screen for small molecules that suppress steroid-inducible and RPM1avrRpml-dependent cell death in Arabidopsis seedlings. Screening of a library comprising 6,800 compounds of natural origin identified two trichothecene-type mycotoxins, 4,15-diacetoxyscirpenol (DAS) and neosolaniol (NEO), which are synthesized by Fusarium and other fungal species. However, protein blot analysis revealed that DAS and NEO inhibit AvrRpm1 synthesis rather than suppress RPM1-
\end{abstract}

Electronic supplementary material The online version of this article (doi:10.1007/s00425-010-1105-1) contains supplementary material, which is available to authorized users.

M. Serrano $\cdot$ P. Schulze-Lefert · E. Kombrink $(\square)$

Department of Plant-Microbe Interactions,

Max Planck Institute for Plant Breeding Research,

Carl-von-Linné-Weg 10, 50829 Cologne, Germany

e-mail: kombrink@mpiz-koeln.mpg.de

D. A. Hubert · J. L. Dangl

Department of Biology, University of North Carolina,

Chapel Hill, NC 27599-3280, USA

Present Address:

M. Serrano

Department of Biology, University of Fribourg,

Chemin du Musée 10, 1700 Fribourg, Switzerland mediated responses. This inhibition of translational activity likely explains the survival of the seedlings under screening conditions. Likewise, flg22-induced defense responses are also impaired at the translational, but not the transcriptional, level by DAS or NEO. Unexpectedly, both compounds not only prevented AvrRpm1 synthesis, but rather caused an apparent hyper-accumulation of RPM1 and HSP70. The hyper-accumulation phenotype is likely unrelated to the ribotoxic function of DAS and NEO and could be due to an inhibitory activity on the proteolytic machinery of Arabidopsis or elicitor-like activities of type A trichothecenes.

Keywords Chemical genetics - Disease resistance . Fusarium mycotoxins - Hypersensitive response . Plant immunity · Trichothecenes
Abbreviations
CHX Cycloheximide
DAS 4,15-Diacetoxyscirpenol
DEX Dexamethasone
ED $\beta$-Estradiol
NEO Neosolaniol

\section{Introduction}

Plants are sessile organisms that need to continuously adjust to environmental changes and therefore evolved elaborate mechanisms to monitor and respond to biotic and abiotic stresses. One example is the plant immune system that enables plants to detect the presence of microbial pathogens and to trigger powerful immune responses. Two classes of plant immune receptors recognize non-self molecular structures (Chisholm et al. 2006; Jones and Dangl 2006). Plasma-membrane-resident pattern recognition receptors 
(PRRs) detect on the cell surface highly conserved microbeor pathogen-associated molecular patterns (MAMPs or PAMPs), such as elicitor-active epitopes of bacterial flagellin (flg22) or fungal chitin, and mount powerful immune responses to non-adapted pathogens (Chisholm et al. 2006; Jones and Dangl 2006; Bittel and Robatzek 2007). Hostadapted pathogens have evolved the means to escape from recognition or suppress MAMP-triggered immunity by delivering effector proteins into host cells of which several are known to intercept PRR-triggered immune signaling (Nomura et al. 2005; Göhre and Robatzek 2008). A second class of immune sensors (also called resistance or $\mathrm{R}$ proteins) acts mainly inside plant cells and recognizes, directly or indirectly, the presence of strain-specific pathogen effectors. Most $\mathrm{R}$ proteins have a tripartite architecture consisting of N-terminal coiled-coil (CC) or Toll/interleukin-1 receptor (TIR) domains, a central nucleotide binding (NB) site, and C-terminal leucine-rich repeats (LRRs) (Shen and Schulze-Lefert 2007). R protein-mediated immunity typically involves defense gene activation and the hypersensitive cell death response (HR) at the site of attempted colonization (Heath 2000; Greenberg and Yao 2004; Stuible and Kombrink 2004).

In Arabidopsis thaliana, Resistance to Pseudomonas maculicula 1 (RPM1) confers immunity against Pseudomonas syringae expressing either of two sequence-unrelated type III effector proteins, AvrRpm1 or AvrB (Grant et al. 1995). Once AvrRpm1 or AvrB is delivered into host cells, the plasma-membrane-associated RPM1-interacting protein (RIN4) is phosphorylated (Mackey et al. 2002). The CC-NBLRR-type protein RPM1 is thought to detect this RIN4 modification and subsequently activates unknown signaling pathway(s) that are tightly linked to defense gene expression and the onset of a HR (Boyes et al. 1998; Mackey et al. 2002).

Conditional expression of AvrRpm1 in transgenic Arabidopsis lines leads to a macroscopically visible RPM1dependent cell death response (Tornero et al. 2002a). A $\beta$-estradiol (ED)-inducible avrRpml expression system was used to identify rpml mutants and genes required for RPM1 function (Tornero et al. 2002b; Hubert et al. 2003). Screening of more than $5 \times 10^{5}$ plants resulted in the isolation of 110 independent mutants that failed to recognize AvrRpm1. These were tentatively assigned to six complementation groups, named loss of recognition to AvrRpm1 (LRA). The largest group comprised $95 \mathrm{rpml}$ alleles and the second largest (lral) 8 recessive alleles of $R A R l$. In addition, two allelic mutations in HSP90.2 that act as non-allelic noncomplementation mutations with $\mathrm{rpml}$ were found (Hubert et al. 2003), as were single alleles of uncharacterized complementation groups (Tornero et al. 2002a). Since RAR1 likely acts as co-chaperone together with SGT1b and cytosolic HSP90.2 to regulate folding of pre-activation RPM1 (Holt et al. 2005), forward genetics has not yet revealed components that become specifically engaged in post-activation signaling or execution of immune responses. If RPM1 post-activation processes consist of redundantly acting components or factors required for cell survival, it will be difficult to identify these by conventional mutagenesis.

Small biologically active molecules that activate or impair a specific target of a signal transduction pathway may offer an alternative to forward genetic approaches, if combined with high-throughput screening technologies and small-molecule libraries (Smukste and Stockwell 2005). In plants, this potential is only beginning to be explored (Blackwell and Zhao 2003; Kaschani and van der Hoorn 2007). This approach can circumvent genetic redundancy if a compound affects all functionally related proteins, such as closely related members of a protein family. This can also avoid lethality because the application of a chemical is conditional and reversible, thereby allowing spatial and temporal control. Finally, dosage-dependent phenotypes can be investigated (Smukste and Stockwell 2005). So far, few chemical genetic screens have been applied to a variety of plant processes, including the gravitropic response, auxin- and brassinosteroid-mediated signaling, plant cell morphogenesis and innate immunity (Armstrong et al. 2004; Surpin et al. 2005; Serrano et al. 2007; Yoneda et al. 2007; Gendron et al. 2008; Knoth et al. 2009).

The small number of genetically defined components required for RPM1 function prompted us to use small molecules for chemical interference of $R$ gene-conditioned processes. By taking advantage of the in planta steroidinducible avrRpml expression system, we sifted through a chemical library to identify compounds that interfere with avrRpm1-RPM1-dependent cell death using a previously established hydroponic culture system for Arabidopsis seedlings in a microtiter plate format (Serrano et al. 2007). This procedure revealed one active molecule as mycotoxin of the trichothecene class produced by Fusarium species (Desjardins 2006). From the analysis of structurally related compounds, we determined that the interference of trichothecenes with RPM1-dependent cell death is likely caused by inhibition of the translation of the AvrRpm1 effector. Although our screen did not uncover components of the AvrRpm1-RPM1-mediated response, we present a new experimental approach, which has potential to identify elusive signaling components acting downstream of RPM1.

\section{Materials and methods}

Plant material and growth conditions

We used transgenic $A$. thaliana lines carrying various gene fusions. Plant line a11, ecotype Columbia (RPM1), harboring the $\beta$-estradiol (ED)-inducible avrRpml expression 
system has been previously described (Tornero et al. 2002a). Plants expressing MYC-tagged RPM1 under control of the native promoter have also been described (Boyes et al. 1998), the line used here contains the transgene in the rpml-3 background (Col-0/rpm1-3/RPM1-MYC) (Mackey et al. 2002). Plants expressing the dexamethasone (DEX)-inducible avrRpml containing the HA epitope were generated by crossing two previously described lines containing MYC-tagged RPMI and HA-tagged avrRpml both in the rpm1-3 background (Boyes et al. 1998; Mackey et al. 2002, 2003) and carried to homozygosity by Eui Hwan Chung and we acknowledge provision of this line (background Col-0/rpm1-3/RPM1-MYC/avrRpm1-HA). The DEX-inducible avrRps4-HA transgenic line has also been published (Mackey et al. 2003), as have the Arabidopsis lines carrying the WRKY29p::GUS, 4CL1p::GUS, or ATL2::GUS reporter genes (Serrano et al. 2007).

Growth conditions and chemical library screening

A chemical library comprising 6,800 organic small molecules of natural origin was obtained from Analyticon Discovery (Potsdam, Germany) with all compounds being dissolved in DMSO (10 mM).

Arabidopsis plants were grown in hydroponic culture as previously described (Serrano et al. 2007). Briefly, 2-4 seeds per well of a 96-well microtiter plate were germinated and cultivated at $24^{\circ} \mathrm{C}$ with constant shaking for 10 days in $0.5 \times$ Murashige and Skoog (MS) medium. To induce AvrRpm1-dependent cell death, the medium was replaced by fresh MS medium containing $10 \mu \mathrm{M} \beta$-estradiol (ED) or $10 \mu \mathrm{M}$ dexamethasone (DEX). In addition, $0.1 \mu \mathrm{L}$ of the compounds dissolved in DMSO was added to give a final concentration of $10 \mu \mathrm{M}$ (in $0.1 \mathrm{~mL}$ MS medium). After $24 \mathrm{~h}$ plants were screened visually for development of cell death/necrosis.

Defense gene activation and GUS expression assay

To induce elicitor/PAMP-dependent immune responses and defense gene expression, plants were treated with MS medium containing $1 \mu \mathrm{M}$ flg22 peptide. Histochemical GUS expression assays were performed as described (Jefferson 1987). Samples were mounted on microscope slides using 50\% glycerol and inspected with a Leica MZFLIII stereomicroscope.

SDS-PAGE and protein blot analysis

Total protein was extracted by grinding plants in $50 \mathrm{mM}$ Tris- $\mathrm{HCl}$ (pH 8.0), $1 \%$ SDS, $1 \mathrm{mM}$ EDTA, $1 \mathrm{mM}$ 2-mercaptoethanol and plant protease inhibitor cocktail added as recommended by the supplier (Sigma,
Deisenhofen, Germany). Proteins were resolved by discontinuous SDS-PAGE (on 12\% gels) as previously described (Kombrink et al. 1988). Proteins were transferred electrophoretically (at $30 \mathrm{~V}, 130 \mathrm{~mA}$ overnight) onto nitrocellulose membranes (Hybond-ECL) and specific proteins detected using the Amersham ECL Plus Western blotting system (GE Healthcare, Munich, Germany) as recommended by the manufacturer. For detection of RPM1-MYC, the monoclonal Myc-antibody 9E10 was used (Boyes et al. 1998), which was provided by University of North Carolina, Lineberger Tissue Culture Facility (Chapel Hill, NC, USA), for AvrRpm1-HA and AvrRps4HA detection the commercial HA antibody 3F10 (Roche Diagnostics, Mannheim, Germany) was used and HPS70 was labeled by a polyclonal antiserum kindly provided by Jane Parker (Max Planck Institute, Cologne, Germany). All sera were used at a dilution of 1:1,000.

Protein was determined according to Bradford (1976) using the Bio-Rad (München, Germany) dye reagent and bovine serum albumin as standard.

RNA isolation and reverse transcription-PCR

Total RNA was isolated from Arabidopsis seedlings using the TRIZOL Reagent ${ }^{\circledR}$ as recommended by the manufacturer (Invitrogen, Karlsruhe, Germany) followed by treatment with DNAse I to remove possible DNA contaminations. Reverse transcription-PCR assays were performed using the SuperScript One-Step RT-PCR System with Platinum Taq DNA polymerase (Invitrogene) and $100 \mathrm{ng}$ RNA (DNA free) from each sample. The following cycling conditions were used for cDNA synthesis and PCR amplification: $30 \mathrm{~min}$ at $50^{\circ} \mathrm{C}, 2 \mathrm{~min}$ at $94^{\circ} \mathrm{C}, 30$ cycles of $30 \mathrm{~s}$ at $94^{\circ} \mathrm{C}$, $45 \mathrm{~s}$ at $54^{\circ} \mathrm{C}, 90 \mathrm{~s}$ at $72^{\circ} \mathrm{C}$, followed by a final extension of $10 \mathrm{~min}$ at $72^{\circ} \mathrm{C}$. The products were separated on $1 \%$ agarose gels. The AvrRpm1 transcript was amplified using the primer pair $5^{\prime}$-ATGGGCTGTGTATCGAGCACT- $3^{\prime}$ and $5^{\prime}$-TCTGAGTCAGACTGAACAGCT- $3^{\prime}$, the transcript of WRKY29 (At4g23550) with the primer pair 5'-ATGG ACGAAGGAGACCTAGAAGC- $3^{\prime}$ and $5^{\prime}$-ACGACGCTC TAATCTCCAGAGAC- $3^{\prime}$, the constitutively expressed ACTIN2 (At3g18780) served as control and was amplified with the primer pair $5^{\prime}$-GCCATCCAAGCTGTTCTCTC- $3^{\prime}$ and 5'-GCTCGTAGTCAACAGCAACAA- $3^{\prime}$.

\section{Results}

Screening for suppressors of AvrRPM1-RPM1dependent cell death

Seedlings of A. thaliana, harboring a conditional avrRpm 1 expression system, were grown in liquid culture media in 
microtiter plates as described (Serrano et al. 2007). After 10 days in continuous light, plant growth was apparent by leaf blade expansion of the first true leaves. When seedlings at this stage were treated with $\beta$-estradiol (ED) to activate avrRpml expression, leaves and cotyledons turned necrotic and yellow within $24 \mathrm{~h}$, a phenotype clearly distinguishable from untreated controls (Fig. 1a). To identify small molecules that interfere with this
AvrRpm1-RPM1-dependent cell death response, we utilized a chemical library comprising 6,800 natural compounds, using a previously described procedure (Serrano et al. 2007). Briefly, 10-day-old seedlings were treated simultaneously with ED $(10 \mu \mathrm{M})$ and each of the library components $(10 \mu \mathrm{M})$, and visually monitored for necrosis after $24 \mathrm{~h}$. Only one compound (AN7-F10) was identified that robustly blocked the HR response (Fig. 1a). This

a

ED::avrRpm1 (+ ED)
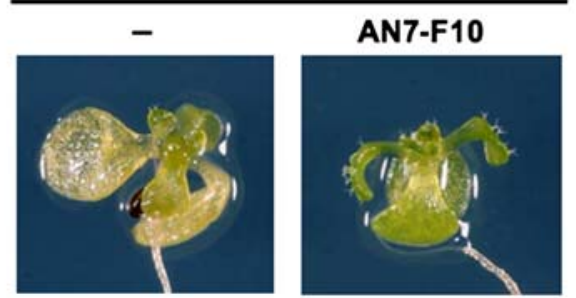

DEX::avrRpm1 (+ DEX)

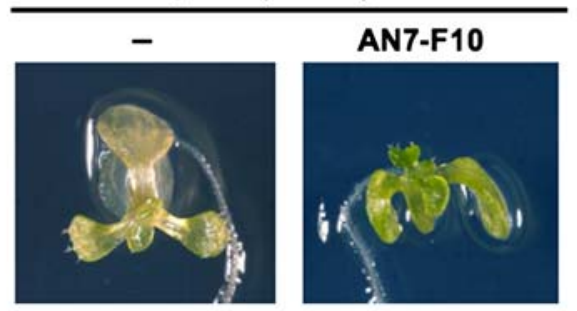

b

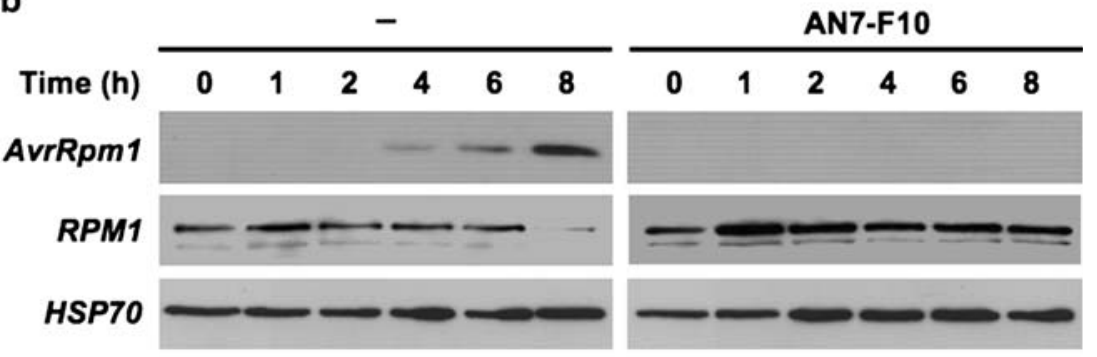

C

$-$

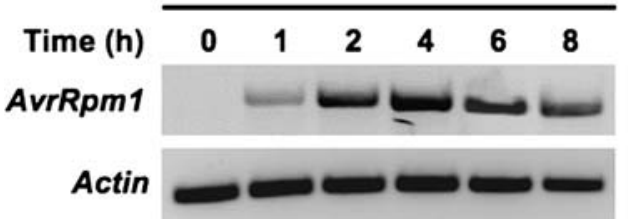

AN7-F10

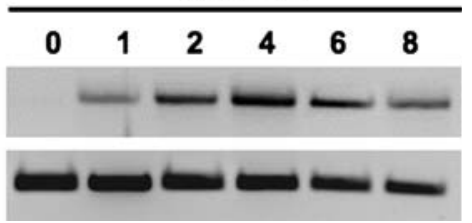

Fig. 1 Screening for small-molecule inhibitors of AvrRpm1-induced cell death in Arabidopsis thaliana. a Phenotype of AvrRpm1-induced necrosis. Ten-day-old transgenic Arabidopsis lines carrying the $\beta$-estradiol (ED)- or dexamethasone (DEX)-inducible avrRpml expression system were treated for $24 \mathrm{~h}$ with $10 \mu \mathrm{M}$ ED or $10 \mu \mathrm{M}$ DEX to induce necrosis (left panels). Simultaneous application of the compound AN7-F10/DAS $(10 \mu \mathrm{M})$ suppressed necrosis formation (right panels). b Quantification of AvrRpm1 and RPM1 by protein blot analysis. Arabidopsis plants (harboring DEX::avrRpm1-HA or ED::avrRpm1 RPM1-MYC) were treated with DEX or ED $(10 \mu \mathrm{M})$ for times indicated. Total protein was extracted and equal aliquots separated by SDS-PAGE and blotted onto nitrocellulose membranes. Specific proteins were detected by anti-HA (AvrRpm1), anti-MYC (RPM1) and anti-HSP70 sera and peroxidase-coupled secondary antibodies, with HSP70 serving as control. One of two independent experiments is shown. $\mathbf{c}$ Quantification of AvrRpm1 mRNA by RTPCR. Total RNA was isolated from 10-day-old Arabidopsis plants that were treated as in b. ACTIN2 (At3g18780) mRNA served as control to normalize transcript levels. Primer pairs for all genes were designed such that they spanned introns and the products were separated on $1 \%$ agarose gels. The experiment was repeated twice 
corresponds to a low hit rate of $0.015 \%$ at stringent screening conditions.

To examine whether AN7-F10 specifically impairs AvrRpm1-triggered cell death, we analyzed, under similar experimental conditions, an additional transgenic Arabidopsis line, in which avrRpml expression is controlled by the dexamethasone (DEX)-activated promoter (Mackey et al. 2003). In this line, AN7-F10 treatment caused a similar cell death inhibition in the presence of DEX, demonstrating that AN7-F10-mediated survival of the plants is independent of the inducible gene expression system used (Fig. 1a).

Delivery of AvrRpm1 or AvrB into plant cells results in RPM1 disappearance and degradation coincident with the onset of the HR response (Boyes et al. 1998). To determine how AN7-F10 exerts its function, we monitored AvrRpm1 and RPM1 amounts in seedlings by protein blot analysis (Fig. 1b). In response to ED or DEX treatment, seedlings started de novo synthesis of AvrRpm1, which was first detectable after $4 \mathrm{~h}$ and resulted in greatly increased steady-state levels by the $8 \mathrm{~h}$ time point. RPM1 amounts declined with a delay to nearly undetectable levels between 6 and $8 \mathrm{~h}$ after ED addition (Fig. 1b, left panel). By contrast, when treatment was carried out in the presence of $10 \mu \mathrm{M}$ AN7-F10, neither AvrRpm1 accumulation nor RPM1 degradation were detectable, indicating that AN7F10 may interfere with AvrRpml expression (Fig. 1b, right panel). Notably, AN7-F10 treatment not only prevented RPM1 degradation but also led to a rapid and sustained increase in RPM1 amounts (Fig. 1b), ranging from 2- to 3fold based on quantitative signal intensities. A similar, although less pronounced increase was also detectable for HSP70 amounts (Fig. 1b). By contrast, ED/DEX-induced accumulation of avrRpm1 transcript was only slightly affected by AN7-F10, suggesting that the compound may selectively impair translation (Fig. 1c).

\section{Fusarium toxins interfere with AvrRPM1-RPM1- dependent cell death}

The structure of the natural compound AN7-F10 was uncovered as 4,15-diacetoxyscirpenol (DAS), a mycotoxin of the trichothecene type, by structural searches in the PubChem database (http://pubchem.ncbi.nlm.nih.gov/). Its structure is shown in Fig. 2a. Trichothecene mycotoxins are secondary metabolites derived from the sesquiterpenoid pathway, which are produced by certain Fusarium strains (Kimura et al. 2007). They exhibit wide range of biological activities and have been classified into four structural types, depending on the presence or absence of certain functional groups (Sudakin 2003). Members of type A include T-2 toxin and DAS (Fig. 2a), which have previously been described to exhibit ribotoxic and elicitor-like activity in a

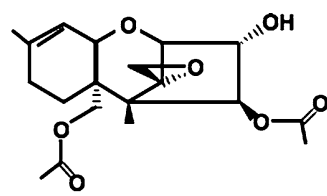

Diacetoxyscirpenol (DAS) AN7-F10

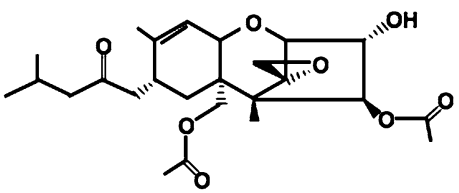

T-2 Toxin

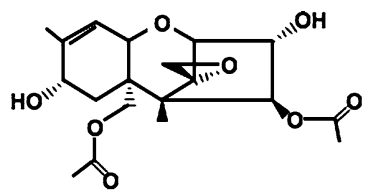

Neosolaniol (NEO) AN2-F4

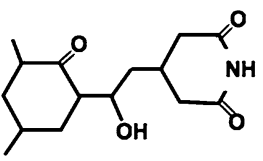

Cycloheximide

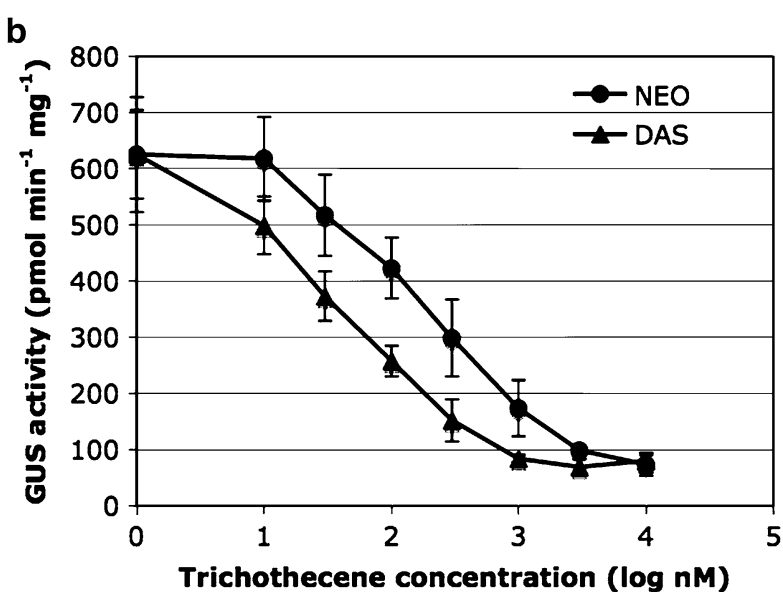

Fig. 2 Chemical structures and biological activity of translation inhibitors. a Structures of selected trichothecenes and cycloheximide. b Inhibition of reporter gene activity by DAS and NEO. The Arabidopsis line harboring WRKY29p::GUS was grown for 10 days in liquid culture; reporter gene expression was induced with flg22 $(1 \mu \mathrm{M}, 4 \mathrm{~h})$ and in parallel plants were treated with different concentrations of DAS or NEO. Specific GUS activity (normalized to protein) of four independent samples $( \pm \mathrm{SD})$ is presented; the derived $\mathrm{IC}_{50}$ values are $50 \mathrm{nM}$ for DAS and $237 \mathrm{nM}$ for $\mathrm{NEO}$

all eukaryotic cells, including animal and plant cells (Rocha et al. 2005; Nishiuchi et al. 2006; Desjardins et al. 2007). The chemical library we used contained an additional type A trichothecene, AN2-F4, which was identified as neosolaniol (NEO) (Fig. 2a). The fact that this compound was not uncovered in the initial screen can be explained by its lower effectiveness in inhibiting RPM1dependent cell death (see below). Using inhibition of reporter gene activity as biological readout, we observed marked differences in the $\mathrm{IC}_{50}$ values for DAS $(50 \mathrm{nM})$ and NEO (237 nM) (Fig. 2b).

DAS and NEO function as inhibitors of protein synthesis

To characterize the impact of DAS and NEO on AvrRPM1-RPM1-mediated responses in more detail, we 
monitored cell death induction as well as AvrRpm1 and RPM1 expression, and compared their effect to that of cycloheximide (CHX), a structurally unrelated inhibitor of translation (Fig. 2a). Clearly, DAS and CHX (each at $10 \mu \mathrm{M})$ delayed AvrRpm1-induced cell death for at least $48 \mathrm{~h}$, whereas NEO $(10 \mu \mathrm{M})$ inhibited necrosis only partially at $24 \mathrm{~h}$ and at later time points yielded a phenotype that was nearly indistinguishable from the control treatment (Fig. 3a). Few studies have directly compared the biological effects of different trichothecene mycotoxins in plants, but in line with our results it has been observed that the activity of trichothecenes differ markedly amongst different structure types (Nishiuchi et al. 2006; Desjardins et al. 2007).

Next, we analyzed the effect of DAS, NEO and CHX on DEX-induced avrRpml expression and RPM1 levels by protein blot analysis (Fig. 3b). DAS and CHX (each at $10 \mu \mathrm{M})$ completely abolished DEX-induced AvrRpm1 accumulation, which in control plants was clearly detectable after 8 and $24 \mathrm{~h}$ of DEX treatment (Fig. 3b). By contrast, in the presence of $10 \mu \mathrm{M}$ NEO AvrRpm1 accumulation was retained at a reduced level after $24 \mathrm{~h}$ that corresponded approximately to the AvrRpm1 amount seen at $8 \mathrm{~h}$ in the absence of this compound (Fig. 3b). Interestingly, despite massive buildup of AvrRpm1 at $24 \mathrm{~h}$ in the presence of DEX, RPM1 steady-state levels were only slightly reduced, if at all (Fig. 3b). Consistent with and extending our previous observation (Fig. 1b), both DAS and NEO-treated plants again showed a 2- to 3-fold increase in RPM1 amounts in comparison to control plants, while HSP70 amounts were only marginally affected. Of note, this increase was not observed in CHX-treated plants (Fig. 3b), suggesting that trichothecene mycotoxins differ from this translational inhibitor by an additional activity that appears to reduce the turnover of preformed proteins (Figs. 1b, 3b).

We examined whether the identified trichothecene inhibitors block conditional in planta accumulation of another bacterial effector, AvrRps4 (Hinsch and Staskawicz 1996). Using a previously characterized Arabidopsis line harboring the DEX-inducible expression system for $a v$ rRps4 (Wirthmueller et al. 2007), the accumulation patterns of the encoded effector in the absence and presence of the translational inhibitors DAS, NEO and CHX, were virtually
Fig. 3 Differential suppression of RPM1-dependent necrosis by protein synthesis inhibitors. a The time course of AvrRpm1induced necrosis is affected by different natural small molecules. In 10-day-old Arabidopsis seedlings (containing DEX::AvrRpm1) avrRpml expression was induced by DEX $(10 \mu \mathrm{M})$; in parallel plants were treated for the times indicated with 4,15diacetoxyscirpenol (DAS), neosolaniol $(N E O)$ or cycloheximide (CHX; $10 \mu \mathrm{M}$ each) and the phenotype scored by microscopy. b Quantification of AvrRpm1, RPM1 and AvrRps4 accumulation by protein blot analysis. Two Arabidopsis lines (harboring DEX::AvrRpm1-HA RPM1$M Y C$ or DEX::AvrRps4-HA) were treated with DEX plus DAS, NEO or CHX $(10 \mu \mathrm{M}$ each) for the time indicated. See Fig $1 \mathrm{~b}$ for experimental details a
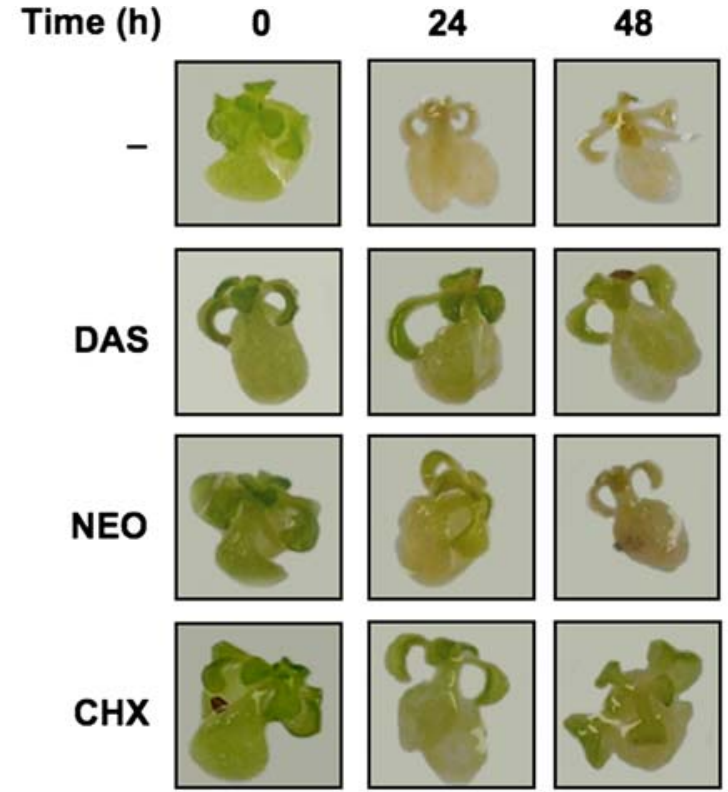

b

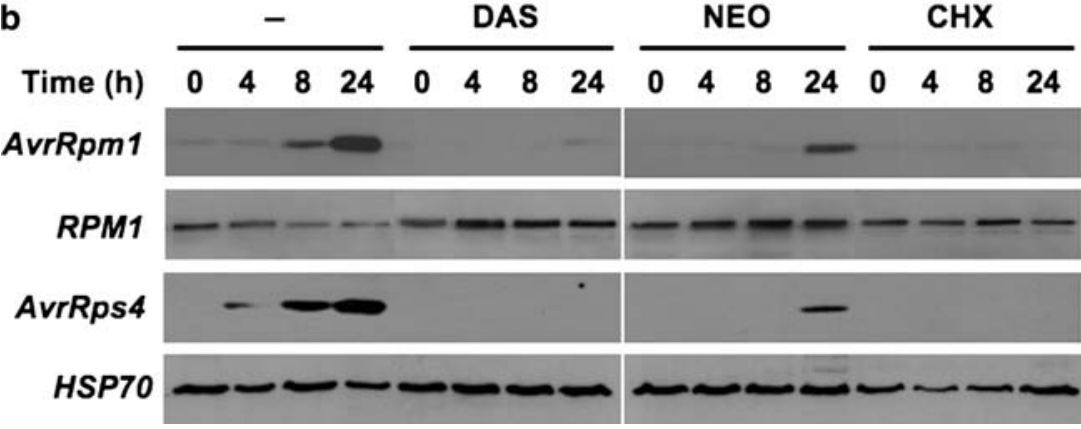


identical to those for AvrRpm1 accumulation (Fig. 3b). Thus, the trichothecene mycotoxins, DAS and NEO, seem to act as general inhibitors of translation, similar to $\mathrm{CHX}$.

DAS and NEO inhibit early MAMP-activated defense responses

By delivering type III effectors, such as AvrRpm1 or AvrRps4, into plant cells, the bacterial pathogen $P$. syringae aims to dampen defense responses triggered by PRR activation (Kim et al. 2005). To determine whether the Fusarium toxins DAS and NEO also affect early MAMPinduced defense responses, we first analyzed a transgenic Arabidopsis line harboring the WRKY29p::GUS reporter gene, which is rapidly activated by the elicitor-active peptide of bacterial flagellin, flg22 (Felix et al. 1999; Gómez-Gómez and Boller 2002; Serrano et al. 2007). In 10-day-old unchallenged plants, expression of WRKY29p:: GUS is confined to leaf primordia and the vasculature, but as early as $2 \mathrm{~h}$ after addition of $1 \mu \mathrm{M}$ flg22, strong expression of the GUS reporter was detectable in leaves, cotyledons and hypocotyl, but not roots of the plant (Fig. 4a). When reporter gene activation by flg22 was monitored in the presence of DAS, NEO or CHX (each at $10 \mu \mathrm{M}$ ), inducible GUS accumulation was below the detection limit (Fig. 4a). To determine whether Fusarium toxins suppress this MAMP-induced defense response at the translational or transcriptional level, we quantified WRKY29 transcripts by RT-PCR (Fig. 4b). In comparison to the control, all flg22-treated samples contained high and comparable amounts of WRKY29 mRNA, thereby corroborating that DAS, NEO and CHX exert their activity through inhibition of de novo protein synthesis without impairing transcription. This demonstrates also that flg22 rapidly activates WRKY29 transcription independent of de novo translation, e.g., by de-stabilization of a repressor or stabilization of an activator. Similar results were obtained with other Arabidopsis lines containing different MAMPresponsive reporter genes, including WRKY22p::GUS, ATL2p::GUS or 4CL1p::GUS (Serrano et al. 2007). In all cases the Fusarium trichothecene toxins impaired flg22induced GUS activity, but not mRNA accumulation (not shown). Finally, using the 4CL1p::GUS reporter line we also demonstrated that DAS and NEO equally impaired translation of the transgene and the endogenous gene by monitoring GUS activity and 4CL1 amounts by protein blot analysis with specific antiserum, respectively (Supplementary Fig. S1).

\section{Discussion}

In this work, we aimed to identify suppressors of a rapid cell death response in hydroponically grown Arabidopsis seedlings, triggered by the intracellular NB-LRR protein RPM1 through recognition of the cognate type III effector AvrRpm1. Most, but not all, known NB-LRR proteininitiated immune responses are associated with a HR that is confined to sites of attempted pathogen colonization (Greenberg and Yao 2004; Stuible and Kombrink 2004).

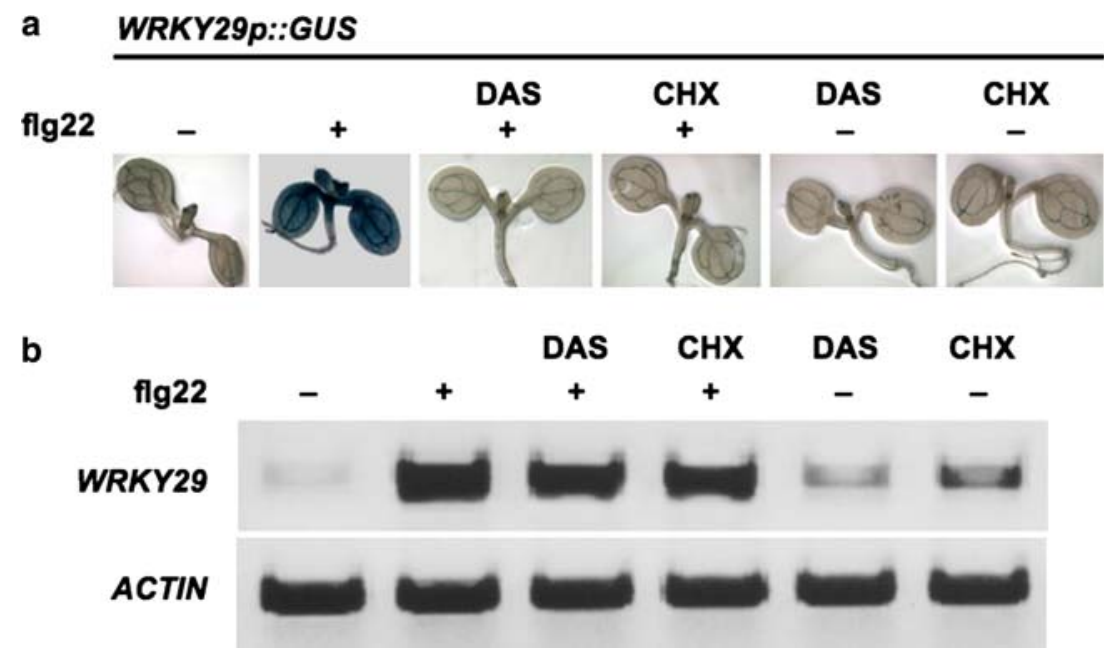

Fig. 4 Chemical inhibition of rapid MAMP-activated gene expression. a Histochemical analysis of GUS activity. In 10-day-old transgenic Arabidopsis lines, homozygous for a single insertion of WRKY29p::GUS, reporter gene expression was induced with flg22 $(1 \mu \mathrm{M}, 2 \mathrm{~h})$; in parallel plants were treated with DAS, NEO or CHX (10 $\mu \mathrm{M}$ each). Representative pictures of three independent experiments are shown. b Quantification of WRKY29 mRNA by RT-PCR.
Total RNA was isolated from 10-day-old Arabidopsis plants (containing WRKY29p::GUS) that were treated with flg22 $(1 \mu \mathrm{M}$, $90 \mathrm{~min})$ plus DAS, NEO or CHX $(10 \mu \mathrm{M}$ each). ACTIN2 (At3g18780) mRNA served as control to normalize transcript levels. Primer pairs for all genes were designed such that they spanned introns and the products were separated on $1 \%$ agarose gels. The experiment was repeated twice 
Although it is still unclear whether the HR is the cause or the consequence of disease resistance, cellular collapse is preceded by the co-ordinate activation of many defense reactions that limit pathogen growth quantitatively and provides a macroscopically visible phenotype. In comparison to plants grown in soil or on agar plates, the miniaturized hydroponic culture system in 96-well microtiter plates allows direct and uniform contact of the whole seedling with the chemicals, thereby minimizing amount and time requirements for efficient uptake and distribution to all tissues and cellular targets. Indeed, ED treatment, which is required for conditional avrRpml expression, resulted in a rapid and macroscopically visible cell death within $24 \mathrm{~h}$ (Fig. 1), whereas in soil-grown plants a macroscopically visible phenotype was usually seen 5 days after ED application (Tornero et al. 2002a).

We screened a chemical library comprising 6,800 natural compounds for the reason that the analyzed chemical space would be limited to potentially bioactive compounds preselected by nature (Kaiser et al. 2008). From this library our initial screen identified only a single compound class that apparently interfered with avrRpml-induced HR cell death, resulting in a low hit rate of $0.015 \%$. Using a similar experimental setup, we identified from the same library nine compounds modulating sucrose stress-induced anthocyanin accumulation, corresponding to a hit rate of $0.13 \%$ (M. Serrano and P. Schulze-Lefert, unpublished data). Other recent chemical screens identified small molecules affecting hormone signaling, gravitropism, cell morphogenesis, membrane trafficking or plant defense gene expression with hit rates ranging from 0.04 to $0.52 \%$ (Armstrong et al. 2004; Surpin et al. 2005; Yoneda et al. 2007; Robert et al. 2008; Knoth et al. 2009). By contrast, a screen for chemicals affecting MAMP-triggered immune responses in Arabidopsis resulted in an unusually high hit rate of $4.2 \%$, which can be explained by the nature of the chemical library comprising selected compounds with established biological activities (Serrano et al. 2007). The remarkably low hit rate of the current screen may have one of the following reasons: (1) RPM1 is engaged in at least two distinct and functionally redundant biochemical activities/pathways leading to cell death activation. Although such scenario remains speculative, the probability to chemically interfere with two activities simultaneously would be low. (2) The onset of the cell death response is hair-trigger operated and threshold dependent, and correspondingly, once initiated the response will run to completion yielding the HR phenotype. This interpretation would be in line with our data. In addition to the identified bioactive trichothecene mycotoxin DAS, the chemical library also contained the structurally related derivative 8-hydroxy DAS, commonly referred to as NEO. However, NEO showed no apparent bioactivity in the initial screen. Targeted and more detailed functional analysis uncovered that this compound is less active in suppressing HR development when compared to DAS, requiring higher concentrations for complete inhibition of translation. Thus, since we used an end-point analysis (cell death) rather than a kinetic readout, the sensitivity of the cell death assay is apparently insufficient to identify weakly active compounds. A more quantitative and robust readout, e.g., reporter gene activation that is tightly correlated with HR development, might help to identify other components required for RPM1 function.

RPM1 is a peripheral membrane protein and has been shown to disappear coincident with the onset of the HR triggered by effectors AvrRpm1, AvrB, AvrRpt2, or AvrRps4 during incompatible interactions (Boyes et al. 1998). This is believed to have a role in controlling the extent of host cell death at sites of attempted bacterial colonization. Interestingly, while RPM1 was essentially undetectable at $8 \mathrm{~h}$ after ED treatment (Fig. 1b), little reduction of its steady-state levels was seen after DEX treatment, even by $24 \mathrm{~h}$, despite comparable hormone-induced AvrRpm1 amounts (Fig. 3b). One possibility is that the kinetics of the cell death response upon ED or DEX treatment are different, resulting in differential RPM1 degradation by a hypothetical proteolytic activity, although other modes of RPM1 removal such as sequestration or precipitation cannot be dismissed. In any event, our finding suggests that RPM1 disappearance is not an intrinsic feature of the RPM1-triggered cell death response, but might reflect a particular sensitivity of this NB-LRR protein to plant and/ or bacterium-derived proteolytic machineries.

The identification of cellular targets of small molecules is normally a time-consuming process. By monitoring RPM1 and AvrRpm1 abundance during the course of the HR-like response, we found that DAS and NEO apparently influence RPM1 degradation and/or synthesis by at least two distinct mechanisms (Fig. 1). Their major activity, inhibition of AvrRpm1 accumulation, is in line with one mode of action of trichothecene mycotoxins in animal cells, efficient inhibition of protein synthesis by binding to the peptidyl transferase binding site on ribosomes (McLaughlin et al. 1977; Sudakin 2003; Rocha et al. 2005). Our observation that DAS and NEO also prevent the translation of various plant defense gene products (GUS reporter), while accumulation of the corresponding mRNAs was not impaired, validates the plant ribosome as one molecular target of trichothecene mycotoxins (Fig. 4). In addition, because translation of AvrRpm1 and AvrRps4 in the presence of DAS and CHX was effectively blocked in our experimental setup (Fig. 3b), the essentially unaltered RPM1 steady-state levels during the $24 \mathrm{~h}$ time course experiment points to a remarkable in planta stability of pre-activation RPM1.

The trichothecenes constitute a family of more than 200 sesquiterpenoid metabolites that are produced by several 
fungal genera, including Fusarium, Myrothecium, Trichoderma, Trichothecium, and others (Grove 2007; Kimura et al. 2007). Their common structural feature comprises a tricyclic skeleton, a 9,10-double bond and a 12,13-epoxide (Fig. 2a). Depending on additional substitutions different structural groups (types A-D) can be distinguished (Sudakin 2003; Kimura et al. 2007). In addition to their toxic effects on all animals, trichothecene mycotoxins are also reported to be toxic to plants, for example, causing growth retardation in wheat and Arabidopsis (Eudes et al. 2000; Poppenberger et al. 2003; Masuda et al. 2007). Different trichothecene chemotypes differ drastically in their toxicity. In a comprehensive study, 22 trichothecenes were tested for their toxicity to A. thaliana in a leaf assay, uncovering more than 200-fold differences in the $\mathrm{LD}_{50}$ (Desjardins et al. 2007). The most potent compounds (displaying $\left.\mathrm{LD}_{50}<10 \mu \mathrm{M}\right)$ included $\mathrm{T}-2$ toxin $(0.5 \mu \mathrm{M}$ ) and DAS $(1.5 \mu \mathrm{M})$, whereas NEO and deoxynivalenol (DON) were active at approximately tenfold higher concentrations [scored at 7 days after application; (Desjardins et al. 2007)]. With respect to HR suppression, we observed a similar difference in bioactivity between DAS and NEO (Fig. 3), and although both compounds were tested at high concentrations $(10 \mu \mathrm{M})$, we did not notice obvious phytotoxic effects. This is presumably due to differences in the duration of treatment; while toxicity was scored after 7 days, we normally evaluated plants after $24 \mathrm{~h}$ when a gross toxic effect had not yet manifested. However, very effective inhibition of reporter gene activity/translation by DAS and NEO was recorded in Arabidopsis seedlings (scored at $4 \mathrm{~h}$ after application) with $\mathrm{IC}_{50}$ values of 50 and 237 nM, respectively (Fig. 2b).

When infiltrated into Arabidopsis leaves, trichothecenes of type A, but not type B, showed elicitor-like activities and caused lesion formation (Nishiuchi et al. 2006). A prolonged activation of mitogen-activated protein kinases (MAPK6 and p44-MAPK) occurred within $1 \mathrm{~h}$, followed by salicylic acid accumulation and expression of defenserelated genes ( $P R-1$ and $P D F 1.2)$ at $48 \mathrm{~h}$, whereas lesion formation, associated with hydrogen peroxide accumulation and callose synthesis, became apparent at 5 days after treatment. Like in animal cells, type A trichothecenes (T2 toxin, DAS) were the most potent translational inhibitors in Arabidopsis protoplasts (80-90\% inhibition of translation at $1 \mu \mathrm{M}$ ), whilst DON (type B) was only slightly inhibitory at this concentration (Nishiuchi et al. 2006). It is unlikely that the inhibition of protein translation by type A trichothecenes is causally linked to their elicitor-like activity and cell poisoning, since $\mathrm{CHX}$, another potent translational inhibitor, neither acted as an elicitor nor induced lesion formation in Arabidopsis (Nishiuchi et al. 2006).

Unexpectedly, DAS and NEO, but not CHX treatment, resulted in an apparent hyper-accumulation of RPM1
(Figs. 1b and 3b). A similar hyper-accumulation pattern, though less pronounced, was found for HSP70. Since each of the compounds inhibits the translational machinery, the DAS or NEO-induced hyper-accumulation effect is likely unrelated to their ribotoxic activities and suggests that both compounds have other targets in plant cells. One possibility is that trichothecenes have additional inhibitory activities on the proteolytic machinery of Arabidopsis cells, resulting in only moderate increases in protein levels because of the concomitant inhibition of translation. However, at present we cannot rule out that the protein hyper-accumulation phenotype is linked to the elicitor-like activities of type A trichothecenes, which include DAS and NEO (Nishiuchi et al. 2006; Kimura et al. 2007). Additional experiments will be required to unravel the precise mode(s) of action of these compounds.

In conclusion, we established a specific and reliable high-throughput screening system for chemical suppressors of the conditional RPM1-mediated cell death response in Arabidopsis. This new experimental approach bears great promise to complement traditional forward genetic screens, which have so far failed to identify components downstream of RPM1 that are involved in execution of immune responses. Small molecules with activating or inhibitory activity may help to dissect biological processes that otherwise suffer from lethal or redundant gene functions. Although our current screen has not resulted in the identification of such compounds, we have established the basic screening conditions as well as ways for improvement. We currently explore alternative reporter-based readouts and more extensive library screening to generate specific chemical tools for dissection of plant defense mechanisms.

Acknowledgments We thank Dr. David Mackey (Ohio State University, Columbus) and Mr. Eui Hwan Chung (University of North Carolina, Chapel Hill) for providing Arabidopsis lines harboring hormone-inducible avrRpml expression systems and Martha Torres for technical assistance. This work was in part supported by Fonds der Chemischen Industrie, the Deutsch-Israelische Projektkoordination (DIP) grant "Chemical genetic platforms for the study of plant biology" to P.S.-L., the Chemical Genomics Centre of the Max Planck Society to M.S. and N.S.F Arabidopsis 2010 grant IOS0520003 to J.L.D. and an NSF Arabidopsis 2010: International Research Experience for Senior Graduate Student award to D.A.H.

Open Access This article is distributed under the terms of the Creative Commons Attribution Noncommercial License which permits any noncommercial use, distribution, and reproduction in any medium, provided the original author(s) and source are credited.

\section{References}

Armstrong JI, Yuan S, Dale JM, Tanner VN, Theologis A (2004) Identification of inhibitors of auxin transcriptional activation by means of chemical genetics in Arabidopsis. Proc Natl Acad Sci USA 101:14978-14983 
Bittel P, Robatzek S (2007) Microbe-associated molecular patterns (MAMPs) probe plant immunity. Curr Opin Plant Biol 10:335341

Blackwell HE, Zhao Y (2003) Chemical genetic approaches to plant biology. Plant Physiol 133:448-455

Boyes DC, Nam J, Dangl JL (1998) The Arabidopsis thaliana RPM1 disease resistance gene product is a peripheral plasma membrane protein that is degraded coincident with the hypersensitive response. Proc Natl Acad Sci USA 95:15849-15854

Bradford MM (1976) A rapid and sensitive method for the quantification of microgram quantities of protein utilizing the principle of protein-dye binding. Anal Biochem 72:248-254

Chisholm ST, Coaker G, Day B, Staskawicz BJ (2006) Host-microbe interactions: shaping the evolution of the plant immune response. Cell 124:803-814

Desjardins AE (2006) Fusarium mycotoxins. Chemistry, genetics and biology. American Phytopathological Society, St. Paul, MN (USA)

Desjardins AE, McCormick SP, Appell M (2007) Structure-activity relationships of trichothecene toxins in an Arabidopsis thaliana leaf assay. J Agric Food Chem 55:6487-6492

Eudes F, Comeau A, Rioux S, Collin J (2000) Phytotoxicité de huit mycotoxines associées àla fusariose de l'épi chez le blé. Can J Plant Pathol 22:286-292

Felix G, Duran JD, Volko S, Boller T (1999) Plants have a sensitive perception system for the most conserved domain of bacterial flagellin. Plant J 18:265-276

Gendron JM, Haque A, Gendron N, Chang T, Asami T, Wang Z-Y (2008) Chemical genetic dissection of brassinosteroid-ethylene interaction. Mol Plant 1:368-379

Göhre V, Robatzek S (2008) Breaking the barriers: microbial effector molecules subvert plant immunity. Annu Rev Phytopathol 46:189-215

Gómez-Gómez L, Boller T (2002) Flagellin perception: a paradigm for innate immunity. Trends Plant Sci 7:251-256

Grant MR, Godiard L, Straube E, Ashfield T, Lewald J, Sattler A, Innes RW, Dangl JL (1995) Structure of the Arabidopsis RPMI gene enabling dual specificity disease resistance. Science 269:843-846

Greenberg JT, Yao N (2004) The role and regulation of programmed cell death in plant-pathogen interactions. Cell Microbiol 6:201211

Grove JF (2007) The trichothecenes and their biochemistry. Fortschr Chem Org Naturst 88:63-130

Heath MC (2000) Hypersensitive response-related death. Plant Mol Biol 44:321-334

Hinsch M, Staskawicz B (1996) Identification of a new Arabidopsis disease resistance locus, RPS4, and cloning of the corresponding avirulence gene, avrRps4, from Pseudomonas syringae pv pisi. Mol Plant Microbe Interact 9:55-61

Holt BF III, Belkhadir Y, Dangl JL (2005) Antagonistic control of disease resistance protein stability in the plant immune system. Science 309:929-932

Hubert DA, Tornero P, Belkhadir Y, Krishna P, Takahashi A, Shirasu K, Dangl JL (2003) Cytosolic HSP90 associates with and modulates the Arabidopsis RPM1 disease resistance protein. EMBO J 22:5679-5689

Jefferson RA (1987) Assaying chimeric genes in plants: the GUS gene fusion system. Plant Mol Biol Rep 5:387-405

Jones JDG, Dangl JL (2006) The plant immune system. Nature 444:323-329

Kaiser M, Wetzel S, Kumar K, Waldmann H (2008) Biology-inspired synthesis of compound libraries. Cell Mol Life Sci 65:1186-1201

Kaschani F, van der Hoorn R (2007) Small molecule approaches in plants. Curr Opin Chem Biol 11:88-98
Kim MG, da Cunha L, McFall AJ, Belkhadir Y, DebRoy S, Dangl JL, Mackey D (2005) Two Pseudomonas syringae type III effectors inhibit RIN4-regulated basal defense in Arabidopsis. Cell 121:749-759

Kimura M, Tokai T, Takahashi-Ando N, Ohsato S, Fujimura M (2007) Molecular and genetic studies of Fusarium trichothecene biosynthesis: pathways, genes, and evolution. Biosci Biotechnol Biochem 71:2105-2123

Knoth C, Salus MS, Girke T, Eulgem T (2009) The synthetic elicitor 3,5-dichloroanthranilic acid induces NPR1-dependent and NPR1-independent mechanisms of disease resistance in Arabidopsis. Plant Physiol 150:333-347

Kombrink E, Schröder M, Hahlbrock K (1988) Several "pathogenesis-related" proteins in potato are 1,3- $\beta$-glucanases and chitinases. Proc Natl Acad Sci USA 85:782-786

Mackey D, Holt BF III, Wiig A, Dangl JL (2002) RIN4 interacts with Pseudomonas syringae type III effector molecules and is required for RPM1-mediated resistance in Arabidopsis. Cell 108:743-754

Mackey D, Belkhadir Y, Alonso JM, Ecker JR, Dangl JL (2003) Arabidopsis RIN4 is a target of the type III virulence effector AvrRpt2 and modulates RPS2-mediated resistance. Cell 112:379-389

Masuda D, Ishida M, Yamaguchi K, Yamaguchi I, Kimura M, Nishiuchi T (2007) Phytotoxic effects of trichothecenes on the growth and morphology of Arabidopsis thaliana. J Exp Bot 58:1617-1626

McLaughlin CS, Vaughan MH, Campbell IM, Wei CM, Stafford ME, Hansen BS (1977) Inhibition of protein synthesis by trichothecenes. In: Rodericks JV, Hesseltine CW, Mehlman MA (eds) Mycotoxins in human and health. Pathotox, Park Forest South, pp 263-275

Nishiuchi T, Masuda D, Nakashita H, Ichimura K, Shinozaki K, Yoshida S, Kimura M, Yamaguchi I, Yamaguchi K (2006) Fusarium phytotoxin trichothecenes have an elicitor-like activity in Arabidopsis thaliana, but the activity differed significantly among their molecular species. Mol Plant Microbe Interact 19:512-520

Nomura K, Melotto M, He S-Y (2005) Suppression of host defense in compatible plant-Pseudomonas syringae interactions. Curr Opin Plant Biol 8:361-368

Poppenberger B, Berthiller F, Lucyshyn D, Sieberer T, Schuhmacher R, Krska R, Kuchler K, Glössl J, Luschnig C, Adam G (2003) Detoxification of the Fusarium mycotoxin deoxynivalenol by a UDP-glucosyltransferase from Arabidopsis thaliana. J Biol Chem 278:47905-47914

Robert S, Chary SN, Drakakaki G, Li S, Yang Z, Raikhel NV, Hicks GR (2008) Endosidin1 defines a compartment involved in endocytosis of the brassinosteroid receptor BRI1 and the auxin transporters PIN2 and AUX1. Proc Natl Acad Sci USA 105:8464-8469

Rocha O, Ansari K, Doohan FM (2005) Effects of trichothecene mycotoxins on eukaryotic cells: a review. Food Addit Contam 22:369-378

Serrano M, Robatzek S, Torres M, Kombrink E, Somssich IE, Robinson M, Schulze-Lefert P (2007) Chemical interference of pathogen-associated molecular pattern-triggered immune responses in Arabidopsis reveals a potential role for fatty-acid synthase type II complex-derived lipid signals. J Biol Chem 282:6803-6811

Shen Q-H, Schulze-Lefert P (2007) Rumble in the nuclear jungle: compartmentalization, trafficking, and nuclear action of plant immune receptors. EMBO J 26:4293-4301

Smukste I, Stockwell BR (2005) Advances in chemical genetics. Annu Rev Genom Human Genet 6:261-286 
Stuible H-P, Kombrink E (2004) The hypersensitive response and its role in disease resistance. In: Punja ZK (ed) Fungal disease resistance in plants-biochemistry, molecular biology, and genetic engineering. Haworth Press, Binghamton, NY, pp 57-92

Sudakin DL (2003) Trichothecenes in the environment: relevance to human health. Toxicol Lett 143:97-107

Surpin M, Rojas-Pierce M, Carter C, Hicks GR, Vasquez J, Raikhel NV (2005) The power of chemical genomics to study the link between endomembrane system components and the gravitropic response. Proc Natl Acad Sci USA 102:4902-4907

Tornero P, Chao RA, Luthin WN, Goff SA, Dangl JL (2002a) Largescale structure-function analysis of the Arabidopsis RPM1 disease resistance protein. Plant Cell 14:435-450
Tornero P, Merritt P, Sadanandom A, Shirasu K, Innes RW, Dangl JL (2002b) RARl and NDRI contribute quantitatively to disease resistance in Arabidopsis, and their relative contributions are dependent on the $R$ gene assayed. Plant Cell 14:1005-1015

Wirthmueller L, Zhang Y, Jones JDG, Parker JE (2007) Nuclear accumulation of the Arabidopsis immune receptor RPS4 is necessary for triggering EDS1-dependent defense. Curr Biol 17:2023-2029

Yoneda A, Higaki T, Kutsuna N, Kondo Y, Osada H, Hasezawa S, Matsui M (2007) Chemical genetic screening identifies a novel inhibitor of parallel alignment of cortical microtubules and cellulose microfibrils. Plant Cell Physiol 48:1393-1403 